\title{
Optimal TCSC placement for congestion management in deregulated power systems using antlion optimization algorithm
}

\author{
Majid Moazzami $^{1}$, Hossein Shahinzadeh ${ }^{2}$, Gevork B. Gharehpetian ${ }^{3}$, Abolfazl Shafiei ${ }^{4}$ \\ ${ }^{1}$ Smart Microgrid Research Center, Najafabad Branch, Islamic Azad University, Najafabad, Iran \\ ${ }^{4}$ Department of Electrical Engineering, Najafabad Branch, Islamic Azad University, Najafabad, Iran \\ ${ }^{2,3}$ Department of Electrical Engineering, Amirkabir University of Technology (Tehran Polytechnic), Tehran, Iran
}

\begin{tabular}{l} 
Article Info \\
\hline Article history: \\
Received Jan 29, 2019 \\
Revised Apr 2, 2019 \\
Accepted Apr 16, 2019 \\
\hline Keywords: \\
Antlion optimization \\
Congestion management \\
FACTS devices \\
Optimal placement \\
TCSC
\end{tabular}

\begin{abstract}
Congestion management is one of the important issues in the deregulated power systems. There are several methods to eliminate congestion. Utilizing FACTS devices is an appropriate option for large-scale and quick control of flows of transmission lines. FACTS devices such as Thyristor Controlled Series Capacitor (TCSC) can help to mitigate the transmitting flow of power in the congested lines, which leads to an increase in the network loading ability as well as reduction of both losses and production costs. Due to the considerably high price of FACTS devices, it is important to determine their optimum location on the network. Accordingly, in this paper, the Antlion optimization algorithm (ALO) has been employed to conduct a congestion management analysis to determine the optimal location for the installation of TCSC, which is simulated on an IEEE 14-bus test system subject to satisfy the constraints of the market environment.
\end{abstract}

Copyright @ 2019 Institute of Advanced Engineering and Science. All rights reserved.

\section{Corresponding Author:}

Majid Moazzami,

Department of Electrical Engineering,

Islamic Azad University of Najafabad,

Daneshgah Blvd, Najafabad, Isfahan, Post code: 8514143131, Iran.

Email: m_moazzami@pel.iaun.ac.ir

\section{INTRODUCTION}

In recent decades, an important structural reform has been made in many power systems, which has changed the power industry from a traditional structure to a restructured modern one. This fundamental change in structure and operational rules have become pervasive very soon throughout the world. The former is called restructuring of power systems, and the latter is called deregulation. In this regard, the generation, transmission, distribution segments and energy services were separated from each other in the first step. Then the generation and distribution sectors were divided into several independent companies which may have governmental or non-governmental ownerships or may be private equities.

Subsequently, each one of the generating and distribution companies was allowed to compete with other companies in the wholesale electricity market to exchange electrical energy as a seller or a buyer. Therefore, the increase in competitiveness of electricity commerce has caused the fair price of electricity which is determined based on the supply and demand trade-off mechanism which provides both sides of the trade with the level of satisfaction. The reduction of generation costs, the improvement in ancillary services quality, and improvement in demand-side satisfaction are other benefits of restructuring in power industry. The transmission network is a major obstacle for the deregulation of the power systems because of two reasons. The first reason is respect to the technical issues, which implies that it is not possible to separate the transmission network like generation or distribution sectors to make it competitive. In addition, the requisite of the existence of a proper competition between power providers in supplying electricity is the fair and not controlled interconnections across the power grid [1-2]. Although the concept of transmission network 
congestion already exists in traditional power systems, the term "congestion" has been raised as the deregulation of the electricity industry has been started. The meaning of congestion is the use of transmission network beyond the permissible operating range. The congestion of transmission lines can prevent suppliers from making a new contract, and cause the impossibility of execution of existing contracts, as well as curtailments, monopoly of prices in some areas, damage to electrical equipment in the system due to unplanned load shedding, price spike, increase in the price of electrical energy in some locations, etc. [3-4].

There are many ways to reduce transmission congestion. The utilization of FACTS devices is one of the most effective ways. Utilizing FACTS devices for congestion management purposes is very useful because the limitation on optimum power flow due to the power transmission constraint is basically removable by the power flow control. TCSC is one of the most utilized kinds of the FACTS devices that can be used to absorb or generate reactive power. TCSC can control the transmission power of the line through affecting on the impedance of the target line. An advantage of using this equipment is its quick installation compared to the construction of a new transmission line. Therefore, the utilization of these devices to eliminate or reduce congestion in the short term is justifiable and sensible.

In recent years, the determination of the size and the optimal location of these devices in the networks have drawn a particular attention to this subject as an optimization problem. Various methods have been proposed for finding the optimal size and location of TCSC regard to its generation capacity, losses, and costs, and various articles have been published in this context. In [5], a sensitivity analysis of the active power flow performance index (PI index) has been used for TCSC and TCPAR optimal locating. According to this method, TCSC should be installed in a line that has the most negative sensitivity factor, and TCPAR should be installed in a line that has the largest value of sensitivity factor so that the installation of FACTS devices in the target line must provide the lowest cost and eliminate the congestion. In [6], an approach is introduced to find the optimal location of TCSC subject to reduce the congestion cost (CC) in a competitive electricity market considering shadow prices. In this paper, the performance index for TCSC placement is a combination of lines' power sensitivity factor and shadow prices. In [7], two options of load shedding and utilizing TCSC have been evaluated to manage congestion in a bilateral based power market.

In [8], the effects of the TCSC on the congestion and prices in an electricity market including bilateral contracts are investigated and a LMP-based approach is used. In [9], a study on the optimal location of the TCSC for congestion management in an electricity market based on sensitivity analysis and considering two goals of reducing the total reactive losses of the system and reducing the active power flow performance index (PI index) has been conducted. In [10], the authors have proposed a new method based on the total FACTS annual income and cost pertaining to TCSC subject to determine its optimal location in order to manage congestion in the restructured electricity markets. In [11], TCSC is utilized in the electricity market to improve the ability of the system to transmit more power. In this paper, the sensitivity analysis is used for TCSC placement. In [12], multi-objective particle swarm optimization algorithm (MOPSO) and sequential quadratic programming (SQP) have been employed with regard to the voltage stability index in optimal locating of FACTS devices for congestion management.

In [13], a new method for the TCSC locating is presented in order to absorb the maximum transmittable power by the loads through the network's branches. In [14], the authors have focused on the transmission cost and improving it by installing the TCSC. In [15-16], an optimization method is proposed to find the best location to install TCSC regard to maximizing the loadability of the Malaysian distribution network based on evolutionary optimization technique. Besides, the increase in the network loading with respect to the implications of installing a series of capacitors is investigated using the particle swarm algorithm based on birds' flock behavior. In [17], the particle swarm optimization algorithm (PSO) has been used to find the optimal value and the optimal location of TCSC and SVC in order to increase the reliability of the system. In [18], the authors have used the bacterial foraging algorithm to optimize FACTS devices. In recent years, new evolutionary algorithms such as bat algorithm (BA) [19], glowworm swarm optimization algorithm (GSO) [20], gravity search algorithm (GSA) [21], gray wolf algorithm (GWO) [22], Shuffled frog leaping algorithm (SFLA) [23], biogeography-based optimization (BBO) algorithm [24], and big bang-big crunch (BBBC) optimization algorithm [25] have been widely used to solve optimization problems in power system operation and market analysis. In the present article, a new method is proposed which is obtained by merging the ALO algorithm and optimal power flow, and this approach is employed to determine the optimal TCSC location. The simulation is executed on IEEE 14-bus test system shows ability and effectiveness of the proposed approach.

Int J Rob \& Autom, Vol. 8, No. 2, June 2019: 77 - 88 


\section{MODELING AND FORMULATION OF TCSC IN OPTIMAL LOAD FLOW EQUATIONS}

\subsection{Static modeling of TCSC}

Figure 1 shows the $\pi$ model of a transmission line that is installed between the bus $i$ and the bus $j$. Assume that the complex voltage at the $i^{\text {th }}$ bus and $\mathrm{j}^{\text {th }}$ bus are defined as $V_{i} \angle \delta_{i}$ and $V_{j} \angle \delta_{j}$ respectively. The active and reactive power transmission from bus $i$ to $j$ is represented in (1) and (2):

$$
\begin{aligned}
& P_{i j}=V_{i}^{2} G_{i j}-V_{i} V_{j}\left(G_{i j} \cos \delta_{i j}+B_{i j} \sin \delta_{i j}\right) \\
& Q_{i j}=-V_{i}^{2}\left(B_{i j}+B_{s h}\right)-V_{i} V_{j}\left(G_{i j} \sin \delta_{i j}+B_{i j} \cos \delta_{i j}\right)
\end{aligned}
$$

Similarly, the active and reactive power transmission from bus $j$ to $i$ bus is shown as (3) and (4):

$$
\begin{aligned}
& P_{j i}=V_{j}^{2} G_{i j}-V_{i} V_{j}\left(G_{i j} \cos \delta_{i j}+B_{i j} \sin \delta_{i j}\right) \\
& Q_{j i}=-V_{j}^{2}\left(B_{i j}+B_{s h}\right)-V_{i} V_{j}\left(G_{i j} \sin \delta_{i j}+B_{i j} \cos \delta_{i j}\right)
\end{aligned}
$$

The transmission line model with incorporating a TCSC that is located between the buses $i$ and $j$ is depicted in Figure 2. In the steady state, TCSC is contemplated as a static reactance with the value of $-j x_{c}$. The active and reactive power transmission from the $i^{\text {th }}$ bus to the $j^{\text {th }}$ bus (and also contrariwise) regard to present of TCSC is modelled as (5) to (8):

$$
\begin{aligned}
& P_{i j}^{C}=V_{i}^{2} G_{i j}^{\prime}-V_{i} V_{j}\left(G_{i j}^{\prime} \cos \delta_{i j}+B_{i j}^{\prime} \sin \delta_{i j}\right) \\
& Q_{i j}^{C}=-V_{i}^{2}\left(B_{i j}^{\prime}+B_{s h}\right)-V_{i} V_{j}\left(G_{i j}^{\prime} \sin \delta_{i j}+B_{i j}^{\prime} \cos \delta_{i j}\right) \\
& P_{i j}^{C}=V_{j}^{2} G_{i j}^{\prime}-V_{i} V_{j}\left(G_{i j}^{\prime} \cos \delta_{i j}+B_{i j}^{\prime} \sin \delta_{i j}\right) \\
& Q_{i j}^{C}=-V_{i}^{2}\left(B_{i j}^{\prime}+B_{s h}\right)+V_{i} V_{j}\left(G_{i j}^{\prime} \sin \delta_{i j}+B_{i j}^{\prime} \cos \delta_{i j}\right)
\end{aligned}
$$

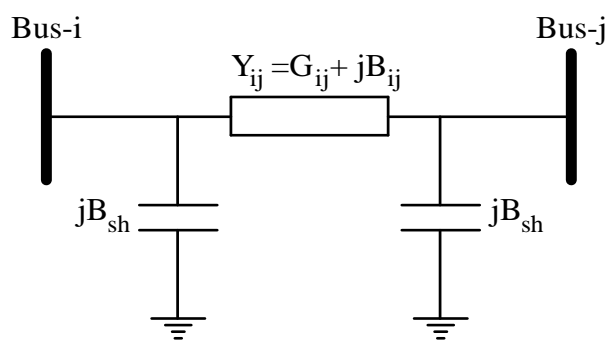

Figure 1. The transmission line model

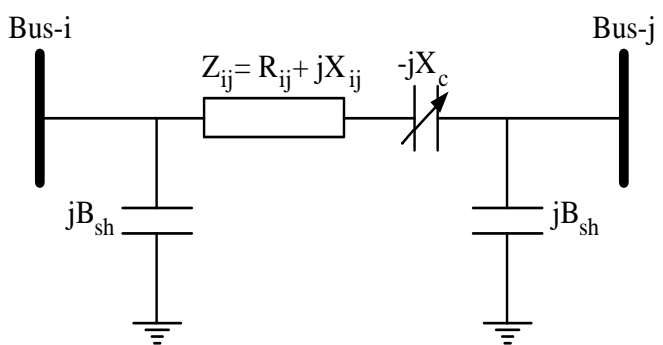

Figure 2. The transmission line model with presence of TCSC

Where $\mathrm{G}_{\mathrm{ij}}^{\prime}$ and $\mathrm{B}_{\mathrm{ij}}^{\prime}$ are (9-10):

$$
\begin{aligned}
& G_{i j}^{\prime}=\left(r_{i j}\right) /\left(r_{i j}^{2}+\left(x_{i j}-x_{C}\right)^{2}\right) \\
& B_{i j}^{\prime}=-\left(x_{i j}-x_{C}\right) /\left(r_{i j}^{2}+\left(x_{i j}-x_{C}\right)^{2}\right)
\end{aligned}
$$

The variation of the line's flow due to series capacitance can be represented as a line without a series capacitance with flowing power at the injecting and receiving terminals of the line, as shown in 
Figure 3. The injected active and reactive power to the bus $i\left(P_{i}{ }^{C}, Q_{i}{ }^{C}\right)$ and to the $j^{\text {th }}$ bus $\left(P_{j}{ }^{C}, Q_{j}{ }^{C}\right)$ can be obtained by (11) to (14) [26-28]:

$$
\begin{aligned}
& P_{i}^{C}=V_{i}^{2} \Delta G_{i j}-V_{i} V_{j}\left(\Delta G_{i j} \cos \delta_{i j}+\Delta B_{i j} \sin \delta_{i j}\right) \\
& P_{j}^{C}=V_{j}^{2} \Delta G_{i j}-V_{i} V_{j}\left(\Delta G_{i j} \cos \delta_{i j}+\Delta B_{i j} \sin \delta_{i j}\right) \\
& Q_{i}^{C}=-V_{i}^{2} \Delta B_{i j}-V_{i} V_{j}\left(\Delta G_{i j} \cos \delta_{i j}-\Delta B_{i j} \sin \delta_{i j}\right) \\
& Q_{j}^{C}=-V_{j}^{2} \Delta B_{i j}+V_{i} V_{j}\left(\Delta G_{i j} \sin \delta_{i j}+\Delta B_{i j} \cos \delta_{i j}\right)
\end{aligned}
$$

Where $\Delta G_{i j}$ and $\Delta B_{i j}$ are (15-16):

$$
\begin{aligned}
& \Delta G_{i j}=\left(x_{C} r_{i j}\left(x_{C}-2 x_{i j}\right)\right) /\left(\left(r_{i j}^{2}+x_{i j}^{2}\right)\left(r_{i j}^{2}+\left(x_{i j}-x_{C}\right)^{2}\right)\right) \\
& \Delta B_{i j}=\left(-x_{C}\left(r_{i j}^{2}-x_{i j}^{2}+x_{C} r_{i j}\right)\right) /\left(\left(r_{i j}^{2}+x_{i j}^{2}\right)\left(r_{i j}^{2}+\left(x_{i j}-x_{C}\right)^{2}\right)\right)
\end{aligned}
$$

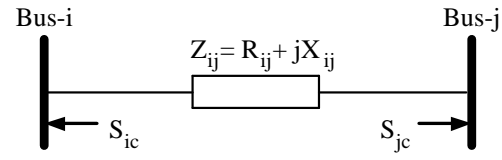

Figure 3. TCSC injection model

\subsection{Optimal load flow equations}

The objective function may contain economic, security or environmental aspects of power systems that must be solved by a proper optimization algorithm. In recent years, regard to the raising of the concept of restructuring and deregulation in the electricity industry, the objective function is mostly defined as the minimization of the generation cost (economic aspects of the system) as well as maximization of the reliability of the system (security of the system).

$$
\mathrm{F}(\mathrm{x})=\min \left(\sum_{i=1}^{N_{G}} c_{i}\left(P_{G_{i}}\right)\right)
$$

Where $F(x)$ is the objective function that must be optimized, $x$ is the state variables, $N_{G}$ is the number of network generators, $c_{i}\left(P_{G i}\right)$ is the generation cost of unit $i$. In general, our goal is to optimize the objective function by a suitable solution and satisfying the prevailing constraints of the system (physical constraints, which limit the power generation and availability of transmission lines' capacities, and the constraints imposed on electrical devices used in power grids and system operational strategies). If the TCSC is located in the line between buses $i$ and $j$, then the power balance equation for the nodes $i$ and $j$ would be expressed as (18-21):

$$
\begin{aligned}
& P_{i}(\theta, V)-P_{G_{i}}+P_{D_{i}}+P_{i}^{T C S C}=0 \\
& Q_{i}(\theta, V)-Q_{G_{i}}+Q_{D_{i}}+Q_{i}^{T C S C}=0 \\
& P_{j}(\theta, V)-P_{G_{j}}+P_{D_{j}}+P_{j}^{T C S C}=0 \\
& Q_{j}(\theta, V)-Q_{G_{j}}+Q_{D_{j}}+Q_{j}^{T C S C}=0
\end{aligned}
$$


Where $P_{G i}$ and $Q_{G i}$ are the active and reactive power in the node $i, P_{G j}$ and $Q_{G j}$ are the active and reactive power in the node $j, P_{D i}$ and $Q_{D i}$ are the active and reactive power consumed by the demand in the node $i, P_{D j}$ and $Q_{D j}$ are the active and reactive power of the loads in the node $j, P_{i}^{T C S C}$ and $Q_{i}^{T C S C}$ are the active and reactive injection power by TCSC to the node $i, P_{j}^{T C S C}$ and $Q_{j}^{T C S C}$ are the active and reactive injection power by TCSC to the node $j$. The constraints of the problem are also expressed in the following (22-26):

$$
\begin{gathered}
\left|S_{i j}(\theta, V) \leq S_{i j}^{\max }\right| \\
P_{G_{i}}^{\min } \leq P_{G_{i}} \leq P_{G_{i}}^{\max } \\
Q_{G_{i}}^{\min } \leq Q_{G_{i}} \leq Q_{G_{i}}^{\max } \\
V_{i}^{\min } \leq V_{G_{i}} \leq V_{i}^{\max } \\
X_{C}^{\min } \leq X_{C} \leq X_{C}^{\max }
\end{gathered}
$$

The (22) shows the limitation of the apparent power through the line where $S_{i j}$ is the apparent passing power through the transmission line between buses $i$ and $j$, and $S_{i j}{ }^{m a x}$ is its maximum boundary. The (23) and (24) explain the active and reactive power generation limitations so that $P_{G i}{ }^{\text {min }}$ and $P_{G i}{ }^{\text {max }}$ imply on the minimum and maximum active power generation boundaries in bus $i, Q_{G i}{ }^{\min }$ and $Q_{G i}{ }^{\max }$ are the minimum and maximum reactive power generation limits in the bus $i$. The (25) shows the voltage range limitation where $V_{i}^{\min }$ and $V_{i}^{\max }$ determine the minimum and maximum limits of the permissible voltage range in the bus $i$. The (26) shows the limitation of the TCSC reactance where $X_{C}{ }^{\min }$ and $X_{C}{ }^{\max }$ are the minimum and maximum of TCSC reactance [29-30]. The optimal power flow optimization problem is formulated as follows:

$$
\left\{\begin{array}{l}
L=\left(\sum_{i=1}^{N_{G}} C_{i}\left(P_{G_{i}}\right)\right)+\sum_{i=1}^{N} \lambda_{P_{i}}\left(P_{i}-P_{G_{i}}+P_{D_{i}}+P_{i}^{T C S C}\right)+\sum_{i=1}^{N} \lambda_{Q_{i}}\left(Q_{i}-Q_{G_{i}}+Q_{D_{i}}+Q_{i}^{T C S C}\right)+\sum_{i j=1}^{N_{L}} \mu_{L_{j i}}\left(S_{i j}-S_{i j}^{\max }\right)+\sum_{i=1}^{N_{G}} \mu_{P_{G_{i}}}^{\min }\left(P_{G_{i}}^{\min }-P_{G_{i}}\right) \\
+\sum_{i j=1}^{N_{G}} \mu_{P_{G_{i}}}^{\max }\left(P_{G_{i}}-P_{G_{i}}^{\max }\right)+\sum_{i=1}^{N_{G}} \mu_{Q_{G_{i}}}^{\min }\left(P_{G_{i}}^{\min }-P_{G_{i}}\right)+\sum_{i=1}^{N_{G}} \mu_{Q_{G_{i}}}^{\max }\left(P_{G_{i}}-P_{G_{i}}^{\max }\right)+\sum_{i=1}^{N} \mu_{V_{i}}^{\min }\left(V_{i}^{\min }-V_{i}\right)+\sum_{i=1}^{N} \mu_{V_{i}}^{\max }\left(V_{i}-V_{i}^{\max }\right)
\end{array}\right.
$$

Where $\lambda$ and $\mu$ denote the Lagrangian coefficients of the equality and inequality constraints respectively, each of which has an economic interpretation. The most important one is $\lambda_{P}$, which is the instantaneous price or nodal price or locational marginal price $(L M P)$. Accordingly, by considering the presence of TCSC in the network, the overall cost function will be made up of two parts:

- The cost of power generating at the plant.

- The cost of investment related to the TCSC.

Thus, the objective function of the system represents the minimization of the cost of generation (economic aspects of the system) and the cost of the TCSC installation:

$$
\mathrm{F}(\mathrm{x})=\min \left(\sum_{i=1}^{N_{G}} C_{i}\left(P_{G_{i}}\right)+C_{T C S C}^{t}\right)
$$

\subsection{Calculation of LMP and congestion analysis}

To calculate the congestion cost in each line, the LMP price difference between two buses must be multiplied by the flow of power passing through the line.

$$
\begin{aligned}
& \Delta \lambda_{i j}=\lambda_{i}-\lambda_{j} \\
& C C_{i j}=\Delta \lambda_{i j} P_{i j}
\end{aligned}
$$


Where $\lambda_{i}$ denotes the locational marginal price in the $i^{\text {th }}$ bus, $\lambda_{j}$ shows the locational marginal price in the $j^{\text {th }}$ bus, $\Delta \lambda_{i j}$ indicates the marginal price difference between buses $i$ and $j, P_{i j}$ represents the flow of passing power through the line $i-j$, and $C_{i j}$ is the congestion cost of the line $i-j$. Accordingly, the total congestion cost can be calculated as in (31) [31-32]:

$$
T C C=\sum_{i j}^{N_{L}} \Delta \lambda_{i j} P_{i j}
$$

\section{ANTLION OPTIMIZATION ALGORITHM}

Antlion optimization $(A L O)$ method is a novel nature-inspired algorithm that is introduced by Mirjalili in 2015 [33]. The $A L O$ algorithm mimics the hunting mechanism of antlions in nature. Five main steps of hunting prey such as the random walk of ants, building traps, entrapment of ants in traps, catching preys, and re-building traps are implemented. There are several different species of ants around the world in nature. Antlions belong to the Myrmeleontidae family and Neuroptera order (net-winged insects). The lifecycle of antlions includes two main phases: larvae and adult. A natural total lifespan can take up to 3 years, which mostly occurs in larvae to become an adult ant. The antlion larvae period mostly passed on walking routes on sand and leaves to find a good place for building traps. During the hunting process, an antlion larva digs a cone-shaped pit in soft sand. As illustrated in Figure 4, after digging the trap, the larvae hide underneath the bottom of the cone, and waits for the prey (ants and other kinds of insects) to be trapped in the pit.

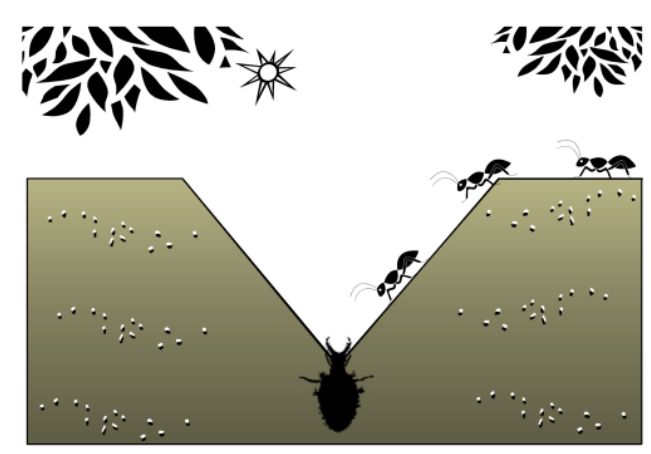

Figure 4. Hunting behavior of antlion

Once the antlion realizes that a prey is in the trap, it tries to catch it. However, if the prey tries to escape from the trap, the antlion intelligently throws sands towards to edge of the pit to slide the prey into the bottom of the pit. The mathematical model of ants and antlions is discussed in the following part:

\subsection{Random walks of ants}

The random walks of ants for searching food in the nature can be expressed by (32):

$$
X(t)=\left[0, \operatorname{cumsum}\left(2 r\left(t_{1}\right)-1\right), \operatorname{cumsum}\left(2 r\left(t_{2}\right)-1\right), \ldots, \operatorname{cumsum}\left(2 r\left(t_{n}\right)-1\right)\right]
$$

Where cum sum is the cumulative sum and $N$ is the maximum number of iteration, $t$ shows the step of random walk and $r(t)$ is a stochastic function defined as (33).

$$
r(t)=\left\{\begin{array}{lll}
1 & \text { if } & \text { rand }>0.5 \\
0 & \text { if } & \text { rand } \leq 0.5
\end{array}\right.
$$

Where $t$ shows the step of random walk and rand is a random number generated in the interval of $[0,1]$. The position of ant is presented in the following matrix: 


$$
M_{A n t}=\left[\begin{array}{cccc}
A_{1,1} & A_{1,2} & \cdots & A_{l, d} \\
A_{2,1} & A_{2,2} & \cdots & A_{2, d} \\
\vdots & \vdots & \ddots & \vdots \\
A_{n, 1} & A_{n, 2} & \cdots & A_{n, d}
\end{array}\right]
$$

Where $M_{\text {Ant }}$ is the matrix for saving the position of each ant, $A_{i, j}$ shows the value of the $j$-th variable of $i$-th ant, $n$ is the number of ants and $d$ is the number of variables. The objective function of each ant is saved in $M_{O A}$ matrix.

$$
M_{O A}=\left[\begin{array}{c}
f\left(\left[A_{1,1}, A_{l, 2}, \ldots, A_{l, d}\right]\right) \\
f\left(\left[A_{2,1}, A_{2,2}, \ldots, A_{2, d}\right]\right) \\
\vdots \\
f\left(\left[A_{n, 1}, A_{n, 2}, \ldots, A_{n, d}\right]\right)
\end{array}\right]
$$

where $f$ denotes the objective function.

$$
M_{\text {Antlion }}=\left[\begin{array}{cccc}
A L_{1,1} & A L_{1,2} & \cdots & A L_{1, d} \\
A L_{2,1} & A L_{2,2} & \cdots & A L_{2, d} \\
\vdots & \vdots & \ddots & \vdots \\
A L_{n, 1} & A L_{n, 2} & \cdots & A L_{n, d}
\end{array}\right]
$$

Where $M_{\text {Antlion }}$ is the matrix for saving the position of each antlion, $A L_{i, j}$ shows the $j$-th value of $i$-th antlion, $n$ is the number of antlions, and $d$ is the number of variables. The objective function of each ant is saved in $M_{O A}$ matrix. Similarly, the objective function of each antlion is saved in $M_{O A L}$ matrix.

$$
M_{O A L}=\left[\begin{array}{c}
f\left(\left[A L_{l, 1}, A L_{l, 2}, \ldots, A L_{l, d}\right]\right) \\
f\left(\left[A L_{2,1}, A L_{2,2}, \ldots, A L_{2, d}\right]\right) \\
\vdots \\
f\left(\left[A L_{n, 1}, A L_{n, 2}, \ldots, A L_{n, d}\right]\right)
\end{array}\right]
$$

In order to keep the random walks inside the search space, a normalizer function is employed shown in (38).

$$
X_{i}^{t}=\frac{\left(X_{i}^{t}-a_{i}\right) \times\left(b_{i}-c_{i}^{t}\right)}{\left(d_{i}^{t}-a_{i}\right)}+c_{i}
$$

Where $a_{i}$ is the minimum of random walk of $i$-th variable, $b_{i}$ is the maximum of random walk in $i$-th variable, $c_{i}^{t}$ is the minimum of $i$-th variable at $t$-th iteration, and $d_{i}^{t}$ indicates the maximum of $i$-th variable at $t$-th iteration.

\subsection{Trapping in antlion's pit}

The mathematical model of the trapped ants in the antlion's traps is presented by (39) and (40).

$$
\begin{gathered}
c_{i}^{t}=\text { Antlion }_{j}^{t}+c^{t} \\
d_{i}^{t}=\text { Antlion }_{j}^{t}+d^{t}
\end{gathered}
$$

Where $c^{t}$ is the minimum of all variables at $t$-th iteration, $d^{t}$ indicates the vector including the maximum of all variables at $t$-th iteration, $c_{j}^{t}$ is the minimum of all variables for $i$-th ant, $d_{j}^{t}$ is the maximum of all variables for $i$-th ant, and Antlion ${ }_{j}^{t}$ shows the selected position of the $j$-th antlion at $t$-th iteration.

Optimal tcsc placement for congestion management in deregulated power systems ... (Majid Moazzami) 


\subsection{Building trap}

In order to model the antlions's hunting capability during the optimization process, a roulette wheel is employed. This mechanism gives high chances to the fitter antlions for catching ants.

\subsection{Sliding ants toward antlion}

With the mechanisms proposed so far, antlions are able to build traps proportional to their fitness and ants are required to move randomly. Moreover, antlions shoot sands outwards the center of the pit to slides down the trapped ant that is trying to escape. Equations (41) and (42) expressed the mathematical model of ants' slide down and trapped in the antlion's trap.

$$
\begin{aligned}
& c^{t}=c^{t} / I \\
& d^{t}=d^{t} / I
\end{aligned}
$$

where $I$ denote the calculated ratio, obtained by (43) [33].

$$
I=10^{w} \cdot(t / T)
$$

Where $t$ is the current iteration, $T$ is the maximum number of iterations, and $w$ is a constant defined based on the current iteration and is obtained by (44).

$$
w=\left\{\begin{array}{lll}
2 & \text { if } & t>0.1 T \\
3 & \text { if } & t>0.5 T \\
4 & \text { if } & t>0.75 T \\
5 & \text { if } & t>0.9 T \\
6 & \text { if } & t>0.95 T
\end{array}\right.
$$

\subsection{Catching prey and re-building the pit}

The final stage of hunt is when an ant (prey) reaches the bottom of the pit and is caught in the antlion's jaw. After this stage, the antlion pulls the prey inside the sand and consumes its body. For mimicking this process, it is assumed that catching prey occur when ants becomes fitter (goes inside sand) than its corresponding antlion. An antlion is then required to update its position to the latest position of the hunted ant to enhance its chance of catching new prey. Equation (45) is proposed in this regard.

$$
\text { Antlion }_{j}^{t}=\text { Ant }_{i}^{t} \quad \text { if } \quad f\left(A n t_{i}^{t}\right)>f
$$

Where $t$ shows the current iteration, Antlion ${ }_{j}^{t}$ shows the position of selected $j$-th antlion at $t$-th iteration, and $A n t_{i}^{t}$ indicates the position of $i$-th ant at $t$-th iteration.

\subsection{Elitism}

Elitism is an important characteristic of evolutionary algorithms that allows the optimization algorithm to select and use the best solution obtained at any stage of optimization process. Since, in optimization process antlion is considered as elite, it should be able to affect the movements of all the ants (preys) during iterations. Therefore, it is assumed that every ant randomly walks around a selected antlion by the roulette wheel and the elite simultaneously. The mathematical model of this behavior is as (46).

$$
A n t_{i}^{t}=\left(R_{A}^{t}+R_{E}^{t}\right) / 2
$$

Where $R_{A}^{t}$ is the random walk around the antlion selected by the roulette wheel at $t$-th iteration, $R_{E}^{t}$ is the random walk around the elite at $t$-th iteration, and $\mathrm{Ant}_{\mathrm{i}}^{\mathrm{t}}$ indicates the position of $i$-th ant at $t$-th iteration $[34,35]$. 


\section{SIMULATION AND RESULTS}

In this paper, IEEE 14-bus test system is used for simulation. This system consists of 5 generators, 11 loads, 17 transmission lines and 3 lines of transformers. The single-line diagram of this network is shown in Figure 5. The information on this network is also given in Tables 1 to 3.

In this method, two cases are investigated:

- TCSC is not installed in the system and the congestion constraint for transmission lines is imposed.

- The lines have congestion constraints and TCSC has been added to the system.

In the first case, in which the system does not have TCSC, the total cost of generation is $8,156.81(\$ / \mathrm{h})$, and the total congestion cost is $1181.32(\$ / \mathrm{h})$. Thus, the total cost will be $9338.13(\$ / \mathrm{h})$. In the second case, after the optimization is executed, the optimal location and size of the TCSC is investigated. The line 2 is determined as the best place to install, and $X_{T C S C}$ is equal to -10.58 . In addition, the total cost of generation in the second case is $8061.46(\$ / \mathrm{h})$, the total congestion cost is $816.32(\$ / \mathrm{h})$. Thus, the total cost will be $8877,78(\$ / \mathrm{h})$. Therefore, with the installation of TCSC in the system, the profit will be equal to $460.35(\$ / \mathrm{h})$. Figure 6 shows the $L M P$ for all buses in the two studied cases. Accordingly, in all buses, aside from buses 3 and 8, the LMP price is reduced. In Figure 7, the transmission power through the network lines is shown in two cases.

Table 1. Cost function coefficients and energy sale biddings

\begin{tabular}{ccccccc}
\hline $\mathrm{P}_{\max }$ & $\mathrm{P}_{\min }$ & Price $(\$ / \mathrm{MWh})$ & $\gamma$ & $\beta$ & $\alpha$ & Bus No. \\
\hline 332 & 0 & 35 & 0.04743 & 20 & 0 & 1 \\
140 & 0 & 36 & 0.2391 & 20 & 0 & 2 \\
100 & 0 & 38 & 0.037 & 35.4 & 0 & 3 \\
100 & 0 & 60 & 0.02 & 40 & 0 & 6 \\
100 & 0 & 40 & 0.03 & 35 & 0 & 8 \\
\hline
\end{tabular}

Table 2. Characteristics of a transmission line

\begin{tabular}{ccccc}
\hline Line No. & Bus $_{\text {Sending }}$ & Bus $_{\text {Receiving }}$ & Resistance (p.u) & Reactance (p.u) \\
\hline 1 & 1 & 2 & 0.01938 & 0.05917 \\
2 & 1 & 5 & 0.05403 & 0.22304 \\
3 & 2 & 3 & 0.04699 & 0.19797 \\
4 & 2 & 4 & 0.05811 & 0.17632 \\
5 & 2 & 5 & 0.05695 & 0.17388 \\
6 & 3 & 4 & 0.06701 & 0.17103 \\
7 & 4 & 5 & 0.01335 & 0.04211 \\
8 & 4 & 7 & 0.00 & 0.20912 \\
9 & 4 & 9 & 0.00 & 0.55618 \\
10 & 5 & 6 & 0.00 & 0.25202 \\
11 & 6 & 11 & 0.09498 & 0.1989 \\
12 & 6 & 12 & 0.12291 & 0.25581 \\
13 & 6 & 13 & 0.06615 & 0.13027 \\
14 & 7 & 8 & 0.00 & 0.17615 \\
15 & 7 & 9 & 0.00 & 0.11001 \\
16 & 9 & 10 & 0.03181 & 0.08450 \\
17 & 9 & 14 & 0.12711 & 0.27038 \\
18 & 10 & 11 & 0.08205 & 0.19207 \\
19 & 12 & 13 & 0.22092 & 0.19988 \\
20 & 13 & 14 & 0.17093 & 0.34802 \\
\hline
\end{tabular}

Table 3. Data of buses [36]

\begin{tabular}{cccccccc}
\hline Bus No. & $\mathrm{P}_{\text {Gen }}$ (p.u) & $\mathrm{Q}_{\text {Gen }}(\mathrm{p} . \mathrm{u})$ & $\mathrm{P}_{\text {Con }}(\mathrm{p} . \mathrm{u})$ & $\mathrm{Q}_{\text {Con }}(\mathrm{p} . \mathrm{u})$ & $\mathrm{Bus}$ type & $\mathrm{Q}_{\text {Max,gen }}$ (p.u $)$ & $\mathrm{Q}_{\text {Min,gen }}(\mathrm{p} . \mathrm{u})$ \\
\hline 1 & 2.32 & 0.00 & 0.00 & 0.00 & $\mathrm{PV}$ & 10.0 & -10.0 \\
2 & 0.4 & -0.424 & 0.2170 & 0.1270 & Swing & 0.5 & -0.4 \\
3 & 0.00 & 0.00 & 0.9420 & 0.1900 & $\mathrm{PV}$ & 0.4 & 0.00 \\
4 & 0.00 & 0.00 & 0.4780 & 0.00 & $\mathrm{PQ}$ & 0.00 & 0.00 \\
5 & 0.00 & 0.00 & 0.0760 & 0.0160 & $\mathrm{PQ}$ & 0.00 & 0.00 \\
6 & 0.00 & 0.00 & 0.1120 & 0.0750 & $\mathrm{PV}$ & 0.24 & -0.06 \\
7 & 0.00 & 0.00 & 0.00 & 0.00 & $\mathrm{PQ}$ & 0.00 & 0.00 \\
8 & 0.00 & 0.00 & 0.00 & 0.00 & $\mathrm{PV}$ & 0.24 & -0.06 \\
9 & 0.00 & 0.00 & 0.2950 & 0.1660 & $\mathrm{PQ}$ & 0.00 & 0.00 \\
10 & 0.00 & 0.00 & 0.0900 & 0.0580 & $\mathrm{PQ}$ & 0.00 & 0.00 \\
11 & 0.00 & 0.00 & 0.0350 & 0.0180 & $\mathrm{PQ}$ & 0.00 & 0.00 \\
12 & 0.00 & 0.00 & 0.0610 & 0.0160 & $\mathrm{PQ}$ & 0.00 & 0.00 \\
13 & 0.00 & 0.00 & 0.1350 & 0.0580 & $\mathrm{PQ}$ & 0.00 & 0.00 \\
14 & 0.00 & 0.00 & 0.1490 & 0.0500 & $\mathrm{PQ}$ & 0.00 & 0.00
\end{tabular}




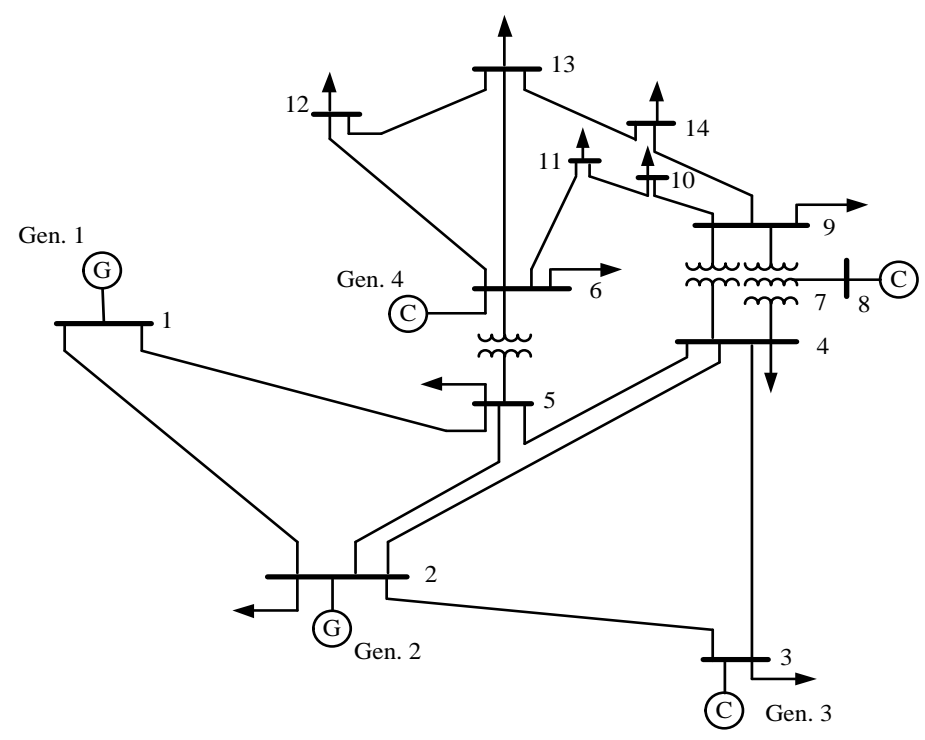

Figure 5. IEEE 14-bus system

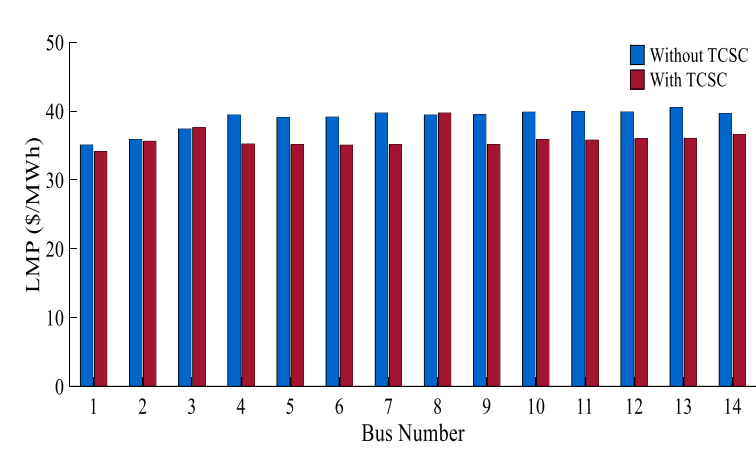

Figure 6. Locational marginal prices with or without TCSC in the IEEE 14-bus test network.

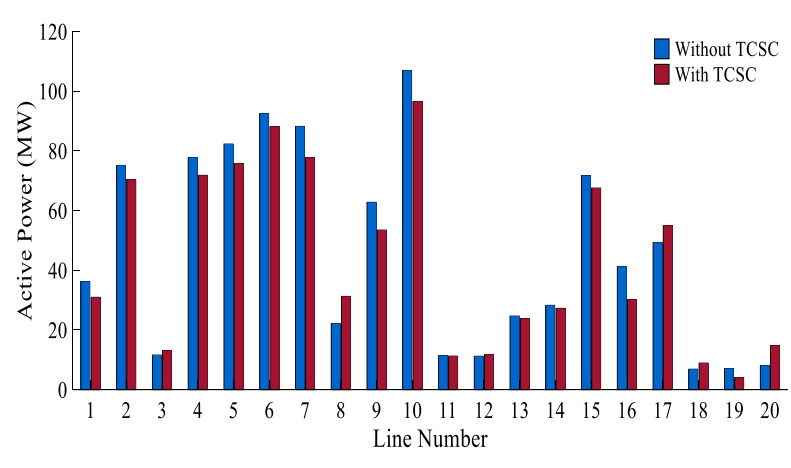

Figure 7. Transmission active power of lines with or without TCSC in the IEEE 14-bus test network

\section{CONCLUSION}

Series FACTS devices can help to increase power system security by controlling the power flow passing through transmission lines. Nevertheless, their considerably high cost of them necessitates the accurate placement and sizing of them. In this study, a combined method is employed to optimize the TCSC parameters and determine its appropriate location to reduce the cost of congestion and to diminish the generation cost in power grid. The result of using ALO algorithm and optimal power flow on an IEEE 14-bus test system is compared in two cases of with and without TCSC. The results show that respect to the optimal installation of TCSC (line 2), the total cost will decrease from $9338.13(\$ / h)$ to $8877.78(\$ / h)$. This means that the profit will be equal to $460.35(\$ / h)$. Therefore, in congestion management method, despite the fact that FACTS devices are expensive, the optimal use of these elements (regard to the best FACTS type selection and choosing the best installation location) mitigates the congestion and make a proper congestion management possible. Therefore, utilizing these devices for congestion management has a higher priority than other methods.

\section{REFERENCES}

[1] FBougouffa, L., \& Chaghi, A., "Optimal Coordination of DOCR for Radial Distribution Systems in Presence of TCSC," International Journal of Power Electronics and Drive Systems, 7(2), 311, 2016.

[2] Kumar, P., "Enhancement of power quality by an application FACTS devices," International Journal of Power Electronics and Drive Systems, 6(1), 10, 2015.

[3] KumariCh, N. R., \& Sekhar, K. C., "Optimal Placement of TCSC Based on Sensitivity Analysis for Congestion Management," International Journal of Electrical and Computer Engineering, 6(5), 2041., 2016. 
[4] Purwoharjono, P., Abdillah, M., Penangsang, O., \& Soeprijanto, A., "Optimal Placement and Sizing of Thyristorcontrolled-series-capacitor using Gravitational Search Algorithm," TELKOMNIKA (Telecommunication Computing Electronics and Control), 10(4), 683-694, 2012.

[5] Singh, S. N., \& David, A. K., "Optimal location of FACTS devices for congestion management," Electric Power Systems Research, 58(2), 71-79, 2001.

[6] Lee, K. H., "Optimal siting of TCSC for reducing congestion cost by using shadow prices," International journal of electrical power \& energy systems, 24(8), 647-653, 2002.

[7] Farahani, V. Z., Kazemi, A., \& Majd, A. B., "Congestion management in bilateral based power market by FACTS devices and load curtailments," In Power India Conference, 2006 IEEE (pp. 6-pp), IEEE, April, 2006.

[8] Acharya, N., \& Mithulananthan, N., "Influence of TCSC on congestion and spot price in electricity market with bilateral contract," Electric power systems research, 77(8), 1010-1018, 2007.

[9] Besharat, H., \& Taher, S. A., "Congestion management by determining optimal location of TCSC in deregulated power systems," International Journal of Electrical Power \& Energy Systems, 30(10), 563-568, 2008.

[10] Afsharnia, S., Ghazizadeh, M. S., \& Pazuki, A., (2008, October), "A new method for optimal location of facts devices in deregulated electricity market," In Electric Power Conference, EPEC 2008, IEEE Canada (pp. 1-6), IEEE, 2008.

[11] Ghawghawe, N. D., \& Thakre, K. L., "Computation of TCSC reactance and suggesting criterion of its location for ATC improvement," International Journal of Electrical Power \& Energy Systems, 31(2), 86-93, 2009.

[12] Wibowo, R. S., Yorino, N., Eghbal, M., Zoka, Y., \& Sasaki, Y., (2009, October), "FACTS devices allocation for congestion management considering voltage stability by means of MOPSO," In Transmission \& Distribution Conference \& Exposition: Asia and Pacific, pp. 1-4, IEEE, 2009.

[13] Duong, T., JianGang, Y., \& Truong, V., "A new method for secured optimal power flow under normal and network contingencies via optimal location of TCSC," International Journal of Electrical Power \& Energy Systems, 52, 68-80, 2013.

[14] Duong, T., JianGang, Y., Nguyen, L., \& ZhenWei, G., "Enhancing Total Transfer Capability via Optimal Location of TCSC in Deregulated Electricity Market. In AETA 2013: Recent Advances in Electrical Engineering and Related Sciences (pp. 47-56)," Springer, Berlin, Heidelberg, 2014.

[15] Wartana, I. M., Singh, J. G., Ongsakul, W., \& Agustini, N. P., (2012, February), “Optimal placement of a series FACTS controller in Java-Bali 24-bus Indonesian system for maximizing system loadability by evolutionary optimization technique," In Intelligent Systems, Modelling and Simulation (ISMS), 2012 Third International Conference on (pp. 516-521). IEEE, 2012.

[16] Wartana, I. M., Singh, J. G., Ongsakul, W., \& Sreedharan, S., "Optimal placement of FACTS controllers for maximising system loadability by PSO," International Journal of Power and Energy Conversion, 4(1), 9-33, 2013.

[17] Mondal, D., Chakrabarti, A., \& Sengupta, A., "Optimal placement and parameter setting of SVC and TCSC using PSO to mitigate small signal stability problem," International Journal of Electrical Power \& Energy Systems, 42(1), 334-340, 2012.

[18] Ali, E. S., \& Abd-Elazim, S. M., "Coordinated design of PSSs and TCSC via bacterial swarm optimization algorithm in a multimachine power system," International journal of electrical power \& energy systems, 36(1), 84-92, 2012.

[19] Ali, E. S., "Optimization of power system stabilizers using BAT search algorithm," International Journal of Electrical Power \& Energy Systems, 61, 683-690, 2014

[20] Shahinzadeh, H., Moazzami, M., Fadaei, D., \& Rafiee-Rad, S. (2017, March), "Glowworm swarm optimization algorithm for solving non-smooth and non-convex economic load dispatch problems," In Fuzzy and Intelligent Systems (CFIS), 2017 5th Iranian Joint Congress on (pp. 103-109), IEEE, 2017.

[21] Shahinzadeh, H., Gheiratmand, A., Fathi, S. H., \& Moradi, J. (2016, April), "Optimal design and management of isolated hybrid renewable energy system (WT/PV/ORES)," In Electrical Power Distribution Networks Conference (EPDC), 2016 21st Conference on (pp. 208-215), IEEE, 2016.

[22] Shakarami, M. R., \& Faraji, I. "Design of SSSC-based Stabilizer to Damp Inter-Area Oscillations Using Gray Wolf Optimization Algorithm," 2015.

[23] Moazzami, M., Gharehpetian, G. B., Shahinzadeh, H., \& Hosseinian, S. H. (2017, March), "Optimal locating and sizing of DG and D-STATCOM using Modified Shuffled Frog Leaping Algorithm," In Swarm Intelligence and Evolutionary Computation (CSIEC), 2017 2nd Conference on (pp. 54-59), IEEE, 2017.

[24] Shahinzadeh, H., Moazzami, M., Abbasi, M., Masoudi, H., \& Sheigani, V. (2016, December), "Smart design and management of hybrid energy structures for isolated systems using biogeography-based optimization algorithm," In Smart Grids Conference (SGC), 2016 (pp. 1-7), IEEE, 2016.

[25] Shahinzadeh, H., Fathi, S. H., Moazzami, M., \& Hosseinian, S. H. (2017, March), "Hybrid Big Bang-Big Crunch Algorithm for solving non-convex Economic Load Dispatch problems," In Swarm Intelligence and Evolutionary Computation (CSIEC), 2017 2nd Conference on (pp. 48-53). IEEE, 2017.

[26] Baghaee, H. R., Kaviani, A. K., Mirsalim, M., \& Gharehpetian, G. B, (2011, May), "Short circuit level and loss reduction by allocating TCSC and UPFC using particle swarm optimization," In Electrical Engineering (ICEE), 2011 19th Iranian Conference on (pp. 1-6). IEEE, 2011.

[27] Baghaee, H. R., Mirsalim, M., Gharehpetian, G. B., \& Kaviani, A. K., "Security/cost-based optimal allocation of multi-type FACTS devices using multi-objective particle swarm optimization," Simulation, 88(8), 999-1010, 2012. 
[28] Sharifi, R., Khamooshi, R., Radmanesh, H., Gharehpetian, G. B., Fathi, S. H., \& Moghani, J. S., "Enhancement of Power System Oscillation Damping using Fuzzy Inference System based Coordinated Design of SSSC Controllers," Indian Journal of Science and Technology, 9(17), 2016.

[29] Jordehi, A. R., "Brainstorm optimisation algorithm (BSOA): An efficient algorithm for finding optimal location and setting of FACTS devices in electric power systems," International Journal of Electrical Power \& Energy Systems, 69, 48-57, 2015.

[30] Ersavas, C., \& Karatepe, E., "Optimum allocation of FACTS devices under load uncertainty based on penalty functions with genetic algorithm," Electrical Engineering, 99(1), 73-84, 2017.

[31] Hooshmand, R. A., Morshed, M. J., \& Parastegari, M., "Congestion management by determining optimal location of series FACTS devices using hybrid bacterial foraging and Nelder-Mead algorithm," Applied Soft Computing, $28,57-68,2015$.

[32] Esmaili, M., Shayanfar, H. A., \& Moslemi, R., "Locating series FACTS devices for multi-objective congestion management improving voltage and transient stability," European journal of operational research, 236(2), 763-773, 2014.

[33] Mirjalili, S., "The ant lion optimizer. Advances in Engineering Software," 83, 80-98, 2015.

[34] Mani, M., Bozorg-Haddad, O., \& Chu, X., “Ant Lion Optimizer (ALO) Algorithm,” In Advanced Optimization by Nature-Inspired Algorithms (pp. 105-116). Springer, Singapore. 2018.

[35] Sanjari, M. J., Fathi, H., Gharehpetian, G. B., \& Tavakoli, A., (2012, May), "HSA-based optimal placement of shunt FACTS devices in the smart grid considering voltage stability," In Smart Grids (ICSG), 2012 2nd Iranian Conference on (pp. 1-6), IEEE, 2012.

[36] Joorabian, M., Saniei, M., \& Sepahvand, H., (2011, June), "Locating and parameters setting of TCSC for congestion management in deregulated electricity market," In Industrial Electronics and Applications (ICIEA), 2011 6th IEEE Conference on (pp. 2185-2190). IEEE., March 2017. 